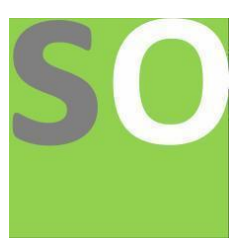

Article title: Immediate loading: principles, requirements, and soft-tissue management

Authors: Vladyslav Pereverzyev[1]

Affiliations: Italy[1]

Orcid ids: 0000-0001-8417-6895[1]

Contact e-mail: lexeto2802@mxcdd.com

License information: This work has been published open access under Creative Commons Attribution License $\mathrm{http}: / / c r e a t i v e c o m m o n s . o r g / l i c e n s e s / b y / 4.0 /$, which permits unrestricted use, distribution, and reproduction in any medium, provided the original work is properly cited. Conditions, terms of use and publishing policy can be found at https://www.scienceopen.com/.

Preprint statement: This article is a preprint and has not been peer-reviewed, under consideration and submitted to ScienceOpen Preprints for open peer review.

DOI: 10.14293/S2199-1006.1.SOR-.PPMD7DR.v1

Preprint first posted online: 12 May 2021

Keywords: Immediate loading, dental implants, implant 


\title{
Immediate loading: principles, requirements, and soft-tissue management
}

\section{Vladyslav Pereverzyev}

\author{
May, 2021
}

\section{Abstract}

Immediate loading is defined as the prosthetic loading of dental implants within the first week of loading. From a biomechanical standpoint, immediate loading can result in success and survival rates comparable to conventionally loaded dental implants. Immediate implant positioning and loading preserved the papillae and soft tissue contours around the implant. Additionally, overall treatment time is decreased and the patient's functional abilities are sooner established. Completing this procedure yields the potential for increased patient satisfaction predictably and efficiently. There are multiple factors to consider in determining whether a patient is an ideal candidate for immediate loading. Placement of an adequate number of implants in well-dispersed positions is necessary in successful immediate loading.

Keywords: Dental implants, Immediate loading,Immediate restoration, Osseointegration, Soft tissue, 
One of the most notable significant scientific advancements in clinical dentistry was undeniably the launch, of osseointegrated implants for anchorage of fixed bridges forty years ago (Albrektsson, Jansson and Lekholm, 1986) (Bobek and Gordon, 2021). Today this is a proven clinical routine with predictable outcomes. Before the introduction of implants, the only care option was to patch damaged teeth with tooth assisted crowns and bridges, or disposable dentures. While fixed devices may be well embraced, not all individuals can adapt effectively to removable dentures but in many situations endure functional and / or emotional issues (Dong et al., 2019).

The advent of osseointegrated implants transformed the science and art of modern dentistry, breathing new life into restorative procedures performed on a daily basis. The recovery of dental implants in the jawbone is centered on the osseointegration theory, which requires a several-month healing duration and is based on establishing direct bone-to-implant contact (BIC), which must be demonstrated histologically (Thalib et al., 2017). As a result, implant dentistry care goals include the predictable creation of a direct bone to implant interface.

Osseointegrated dental implants are placed traditionally, following a two-stage protocol. The 2-stage surgical protocol established by Branemark et al (Brånemark et al., 1977) includes:

(1) Positioning and placement of the implant below the crestal bone to allow for stress healing without loading,

(2) A soft-tissue covering over the implant to be obtained and maintained for 3 to 6 months, and

(3) A minimally loaded implant environment to be maintained for 3 to 6 months.

After this procedure, a second-stage surgery was necessary to uncover these implants and place a prosthetic abutment. Hence, according to the Brånemark protocol (Bra-nemark et al., 1986), dental implants, regardless of their design or system, an undisturbed healing period of three months is required in the mandible and six months in the maxilla. While these periods purportedly allowed time for osseointegration of the implants within the respective arch, but this two stage protocol involved certain disadvantages i.e. it made the implant treatment lengthy. In patients who were either completely or partially edentulous a long term clinical rigid fixation had been reported after this protocol.

Dental implants with osseointegration are typically put in a two-stage procedure. Branemark et al. (Brånemark et al., 1977) developed a two-stage surgical procedure that includes:

(1) Locating and placing of the implant underneath the crestal bone to enable for stress curing without packing,

(2) Obtaining and maintaining a soft-tissue shielding over the implant for three to six months.

(3) A marginally loaded implant setting that will be retained for a period of three to six months. 
A second stage of surgery was needed to extract these implants and install a prosthetic abutment following this procedure. Thus, the Brnemark protocol (Bra-nemark et al., 1986) needs an uninterrupted healing time of three months in the mandible and 6 months in the maxilla for dental implants, regardless of their design or method. While these time periods were intended to allow for osseointegration of the implants inside the respective arch, this two-stage procedure had some drawbacks, namely that it prolonged the implant treatment. In patients who were fully or partially edentulous, this protocol resulted in long-term clinical rigid fixation (Kass et al., 1997) (van Steenberghe et al., 1990).

Additionally, Brnemark's guidelines for healing were focused on observational evidence that was never scientifically validated or ascertained experimentally. Nowadays, with an everincreasing demand for esthetics, the interim duration of edentulousness following implant placement may result in psychological, social, or functional complications, especially if the edentulous area is in the appearance zone. Additionally, the pain, inconvenience, and anxiety associated with such a lengthy wait time continue to be a source of frustration for both patients and clinicians (Cochran, 2006). It has been stated in the scientific literature that root-form implants can osseointegrate even when they extend above the bone and through soft tissues throughout early bone remodeling (Mahesh, Kumar and Babu, 2014) (Douglas De Oliveira et $a l ., 2016)$. This surgical technique is referred to as a one-stage or nonsubmerged implant procedure because it eliminates the need for subsequent implant uncovery surgery. As a result, the pain, inconvenience, and appointments associated with surgery and suture removal are eliminated.

As a result, immediate loading of dental implants was established to overcome the limitations of the original Branemark protocol. Immediate loading implants are those that incorporate a prosthetic superstructure that allows occlusal contact during the first one or two days of placement. Additionally, it can be described as a situation in which the superstructure is attached to the implants within 72 hours of surgery. Not only does it not include underwater one-stage surgery, but it also involves loading the implant without jeopardizing osseointegration. When the occlusion is restored within two weeks, it is referred to as an early loading implant; however, when loading is permitted after several weeks, it is referred to as a delayed loading implant, regardless of whether it is a one- or two-stage operation (Tettamanti et al., 2017) (Zhang, Wang and Song, 2017) (Douglas De Oliveira et al., 2016).

As early as 1979, effective immediate loading of screw-type dental implants was recorded under these conditions. Later, several clinical and laboratory trials by (Chiapasco et al., 2001) (Schnitman et al., 1997); Schnitman et al.21; and Tarnow et al. confirmed the patients' prospects for anticipated dental recovery. These experiments eventually resulted in the development of the principle of immediate loading. Eliminating micro movements between implants and osteotomies is a critical component of immediate occlusal loading protocols. The ultimate aim of an immediate loading protocol is to ideally minimize surgical interventions and the time interval between surgery and prosthesis fulfillment, while maintaining the rates of success associated with implant care and the unloaded healing protocol.

\section{Loading and its Classification}

Loading a dental implant refers to the process of attaching the prosthetic to the implant hardware after it has healed. Early loading, on the other hand, is gaining popularity as patients 
seek shorter treatment times. This means that the implant will be loaded before the tissues have healed completely and the implant has osseointegrated completely (Gapski et al., 2003) (Merli et al., 2020).

There are three distinct loading methods:

i) Conventional,

ii) Early, and

iii) Immediate.

\section{Conventional Loading}

Conventional loading is known as the restoration of prosthetic function and operational loading of an osseointegrated implant following a three- to six-month healing period. As previously mentioned, this protocol was developed for implants with machined surfaces.

Implants are often, but not always, inserted using the conventional loading procedure and then the surgical site is closed, necessitating a second stage operation to "uncover" the implant. This is sometimes referred to as delayed loading (Hong and Oh, 2017) (Alfadda et al., 2019).

\section{Immediate Loading}

Immediate implant loading is on the other end of the continuum. Immediate loading is characterized as re-establishing occlusal contact with the implant within 48 hours of placement. Taken to its logical conclusion, the instantly loaded implant could be mounted and permanently restored in less than 48 hours (Del Fabbro et al., 2015).

Immediate loading has significantly reduced the time required between implant installation and restoration. The patient benefits from a shorter total recovery time, less clinician visits, increased relaxation during the healing process, and enhanced esthetic and phonetic dimensions.

\section{Immediate Restoration}

Immediate restoration or immediate provisionalization are terms that are synonymous with immediate loading. Within 48 hours, the implant is repaired, but in this situation, the repair is left free of any usable occlusion (Glauser, Zembic and Hämmerle, 2006) (Al-Sawai and Labib, 2016).

It is immediate to emphasize that immediate loading and restoration are distinct from immediate implant placement. While often defined in conjunction and undoubtedly connected, implant placement and loading protocols should be treated separately when treating partially and fully edentulous patients. 


\section{Early Loading}

Early loading occurs between conventional and immediate loading in terms of loading. Early loading is described as the loading or utilization of a prosthetic device or implant prior to immediate or conventional loading (Pigozzo et al., 2018).

Six to eight weeks of healing time is typical for early loading protocols, but our understanding of the biologic processes underlying osseointegration and ongoing advancements in implant surface technology continue to reduce the time needed for adequate healing (Xu et al., 2014).

In summary, conventional loading of a dental implant occurs between three and six months after the implant hardware is mounted. Extended treatment periods, on the other hand, can be unfavorable for certain patients, especially when the implant is placed in the front of the mouth. Immediate loading occurs approximately 48 hours after implant hardware placement; however, studies indicate that immediately loaded implants perform less well than conventional implants. The term "Early loading" refers to loading that happens between 48 hours and three to six months.

\section{The immediate loading principle}

When a balanced load is applied to the bone with the aid of the implant, the bone responds by re-modeling its architecture in response to the magnitude and direction of the applied load. Frost's mechanostat theory describes how bone adapts to various biologic processes (Misch, Horn Lay Wang, et al., 2004):
a) Trivial,
b) Physiological,
c) Overload and
d) Pathological.

Re-modelling is a simultaneous forming and resorption mechanism that replaces preexisting bone, helps to eliminate or conserve bone, and is triggered by reduced mechanical use in the trivial/insignificant loading zone or micro disruption in the pathological loading zone.

The primary goal of an immediately loaded implant prosthesis is to minimize the possibility of occlusal overload, thus increasing bone remodeling. The two kinds of bone that shape at the interface are woven and lamellar. Woven bone forms in response to abnormal loading conditions at a rate of more than 60 microns per day and is found to be less mineralized than lamellar bone, which forms at a rate of 1-5 microns per day. As a result, increased turnover rates result in an increased risk of infection at the bone-implant interface. 
The bone level of the extraction site and the adjacent soft tissue volume will decline as teeth are lost, with substantial tissue loss in the first month and continuing for at least six months. Because of the missing soft and hard tissues, shorter implants may be positioned and longer crowns can be built in the healed sites due to reduced bone height. This short-implant/longcrown combination can experience mechanical issues, leading to bone resorption and implant failure. Interdental papillae will be lost when soft tissue is lost. Interdental papillae loss can also affect the esthetic configuration- "black triangle" areas between the crowns that affect the esthetic dental harmony. Phonetic issues and food impaction can also be caused by a lack of interdental papillae (Misch, Hom Lay Wang, et al., 2004).

Immediate implant placement has a range of advantages, including a shorter recovery period, less procedures, and a lower risk of hard and soft tissue resorption (Refaie et al., 2020). In other words, immediate implant placement at extraction sites has been shown to maintain bone and soft tissue thickness. Main stability can be seen in the situations, and an immediate loading procedure can be used in the anterior sites.

Juodzbalys and Wang, (2007) implanted titanium implants into extraction sockets and monitored them for a year. They discovered that the implant success rate was $100 \%$ and that the keratinized soft tissue volume was 92.9 percent adequate.

Immediate implant placement and provisional crown delivery on the same day of surgery provide patients with immediate comfort, hold their teeth in place, and support their lips. Immediate implant positioning and loading assist the hard and soft dental tissues at the extraction sites after extraction. During recovery, provisional crowns preserve and reshape the soft tissue contours (Al-Harbi and Edgin, 2007).

Immediate implant loading in the anterior maxilla protects the interdental papillae and strengthens the soft tissue contours, according to Di Alberti et al., (2012). .Another research found that immediate implant positioning and loading preserved the papillae and soft tissue contours around the implant (Tarnow, Emtiaz and Classi, 1997). However, it is well understood that the region underneath the pontic, which is based on a convex form, can be strengthened by conditioning and imposing positive pressure.

\section{Immediate Loading Requirements}

\section{a) Patient Selection}

When deciding if a patient is a good candidate for immediate loading, there are many factors to consider. Immediate loading might not be a good choice if you have a medical background that includes bisphosphonates, irradiation, or immunosuppression. These patients have a harder time healing wounds and have a higher risk of implant failure. Tobacco use and controlled diabetes, on the other hand, do not inherently pose a risk. There are mental and dental factors to address in addition to medical factors. A past record of periodontitis or parafunctional behaviors aren't always a no-no. However, surgical factors such as implant position, 
bone quality and force distribution may have a negative effect on implant stability. As a result, in addition to occlusal layout for work, prosthetic design must be considered for optimum rigidity of the prosthesis (Huynh-Ba, Oates and Williams, 2018). Adherence to a soft diet and oral hygiene maintenance after surgery are both important factors in a positive outcome.

\section{b) Surgical Factors to Consider}

In order to achieve effective immediate loading, a sufficient number of implants must be placed in well-distributed locations. The aim of implant numbering and positioning is to restrict micromotion and ensure that occlusal forces are distributed evenly. Bone quality, injection torque at the time of surgery, and even implant duration can all affect the number and placement of implants (Iijima et al., 2013). A minimum of four implants in the mandible and 4 to 6 implants in the maxilla is recommended; however, fewer implants may result in unpredictable results. Prosthetic failure can occur as a result of a large cantilever if the distance between implants is too great; conversely, too little distance can reduce resin bulk, resulting in a weaker prosthesis.

\section{C) Restorative consideration}

The ideal prosthesis for full-arch immediate loading is hygienic, robust, and attractive, and it distributes forces evenly. Restorations should be convex to avoid interfering with recovery process and to make hygiene maintenance easier. Screw retention is also advised to prevent cement-related irritation and excessive pull-out forces (Atieh et al., 2009). Occlusal design and allocation of adequate space for teeth and acrylic bulk will accommodate aesthetics, rigidity, and force distribution. Given that survival rates are usually enhanced when immediately loaded prostheses challenging natural dentition have greater success rates than opposing implants, force distribution is critical. If occlusal forces are an issue, flattening cusps for balance and narrowing occlusal surfaces should alleviate the problem.

\section{Conclusion}

The last decade has seen a profound shift in implant dentistry from the lengthy healing original protocols to immediate loading has demanded a cognitive revolution. Immediate loading refers to the placement of prosthetics on dental implants within the first week of placement. Immediate loading can achieve success and survival rates comparable to conventionally loaded dental implants from a biomechanical perspective. The papillae and soft tissue contours around the implant were preserved due to immediate implant positioning and loading. Furthermore, the average recovery period is reduced, and the patient's functional abilities are developed earlier. When this process is completed, it has the ability to improve patient satisfaction in a predictable and effective manner. When deciding if a patient is a good candidate for immediate loading, there are many factors to consider. In order to achieve effective immediate loading, a sufficient number of implants must be placed in well-distributed locations. Immediate loading of a dental implant consists of a nonsubmerged 1-stage surgery in which loading of the implant 
is done with a provisional restoration at the same appointment or shortly thereafter. Immediate loading of dental implants are proven to reduce the treatment time and thus increase patient acceptance. Future studies should be conducted to evaluate long-term data of immediate restorations on implants and possible applications of this technique in situations where problems of poor bone quality, multiple implants or augmentation procedures must be overcome.

\section{References}

Al-Harbi, S. A. and Edgin, W. A. (2007) 'Preservation of soft tissue contours with immediate screwretained provisional implant crown', Journal of Prosthetic Dentistry. doi: 10.1016/S00223913(07)60107-3.

Al-Sawai, A. A. and Labib, H. (2016) 'Success of immediate loading implants compared to conventionally-loaded implants: a literature review', Journal of investigative and clinical dentistry. doi:10.1111/jicd.12152.

Di Alberti, L. et al. (2012) 'Clinical and radiologic evaluation of 70 immediately loaded single implants in the maxillary esthetic zone: preliminary results after 1 year of functional loading.' International Journal of Oral \& Maxillofaciallmplants, 27(1).

Albrektsson, T., Jansson, T. and Lekholm, U. (1986) 'Osseointegrated dental implants.', Dentalclinics of North America. doi: 10.1007/978-1-59259-197-8_5.

Alfadda, S. A. et al. (2019) 'Immediate versus conventional loading of mandibular implant-supported fixed prostheses in edentulous patients: 10 -year report of a randomised controlled trial.', Internationaljournal of oral implantology (New Malden, London, England).

Atieh, M. A. et al. (2009) 'Immediate restoration/loading of immediately placed single implants: Is it an effective bimodal approach?: Review', ClinicalOral Implants Research. doi:10.1111/j.16000501.2009.01725.x.

Bobek, T. and Gordon, A. (2021) 'A review of the determinants of a successful endosseous implants'. OSF Preprints.

Bra-nemark, P.-I. et al. (1986) 'Tissue-integrated prostheses. osseointegration in clinical dentistry'. LWW.

Brånemark, P. I. et al. (1977) 'Osseointegrated implants in the treatment of the edentulous jaw. Experience from a 10-year period.' , Scandinavian journal of plastic and reconstructive surgery. Supplementum.

Chiapasco, M. et al. (2001) 'Implant-retained mandibular overdentures with Brånemark System MKII implants: a prospective comparative study between delayed and immediate loading.' , International Journalof Oral \& Maxillofaciallmplants, 16(4).

Cochran, D. L. (2006) 'The Evidence for Immediate Loading of Implants', Journal of Evidence-Based Dental Practice. doi:10.1016/j.jebdp.2006.04.018.

Dong, H. et al. (2019) 'Satisfaction analysis of patients with single implant treatments based on a questionnaire survey', Patient Preference and Adherence. doi: 10.2147/PPA.S201088.

Douglas De Oliveira, D. W. et al. (2016) 'Dental Implants with Immediate Loading Using Insertion Torque of $30 \mathrm{Ncm}$ : A Systematic Review', Implant Dentistry. doi: 10.1097/ID.0000000000000444. 
Del Fabbro, M. et al. (2015) 'Immediateloading of postextraction implants in the esthetic area: Systematic review of the literature', Clinical Implant Dentistry and Related Research . doi: 10.1111/cid.12074.

Gapski, R. et al. (2003) 'Critical review of immediate implant loading', Clinical Oral Implants Research. doi:10.1034/j.1600-0501.2003.00950.x.

Glauser, R., Zembic, A. and Hämmerle, C. H. F. (2006) 'A systematic review of marginal soft tissue at implants subjected to immediate loading or immediate restoration', Clinical Oral Implants Research. doi:10.1111/j.1600-0501.2006.01355.x.

Hong, D. G. K. and Oh, J. (2017) 'Recent advances in dental implants', Maxillofacial Plastic and Reconstructive Surgery. doi: 10.1186/s40902-017-0132-2.

Huynh-Ba, G., Oates, T. W. and Williams, M. A. H. (2018) 'Immediate loading vs. early/conventional loading of immediately placed implants in partially edentulous patients from the patients' perspective: A systematic review', Clinical Oral Implants Research. doi: 10.1111/clr.13278.

lijima, M. et al. (2013) 'Effect of immediate loading on the biomechanical properties of bone surrounding the miniscrewimplants', European Journal of Orthodontics. doi: 10.1093/ejo/cjs055.

Juodzbalys, G. and Wang, H. L. (2007) 'Soft and hard tissue assessment of immediate implant placement: A case series', Clinical Oral Implants Research. doi: 10.1111/j.1600-0501.2006.01312.x.

Kass, R. E. et al. (1997) 'Markov Chain Monte Carlo in Practice.' , Journal of the American Statistical Association. doi: 10.2307/2965438.

Mahesh, P., Kumar, V. C. and Babu, K. S. (2014) 'Immediate loading of dental implants: a review-Part I', Annals and Essences of Dentistry. doi: 10.5958/0976-156x.2014.00024.0.

Merli, Mauro et al. (2020) 'Immediate versus early non-occlusal loading of dental implants placed flapless in partially edentulous patients: A 10-year randomized clinical trial', Journal of Clinical Periodontology. doi: 10.1111/jcpe.13279.

Misch, C. E., Wang, Horn Lay, et al. (2004) 'Rationale for the application of immediate load in implant dentistry: Part l', Implant Dentistry. doi:10.1097/01.id.0000140461.25451.31.

Misch, C. E., Wang, Hom Lay, et al. (2004) 'Rationale for the application of immediate load in implant dentistry: Part II', Implant Dentistry. doi: 10.1097/01.id.0000148556.73137.24.

Pigozzo, M. N. et al. (2018) 'Immediate versus early loading of single dental implants: A systematic review and meta-analysis', Journal of Prosthetic Dentistry. doi: 10.1016/j.prosdent.2017.12.006.

Refaie, A. et al. (2020) 'Effect of different immediate implant loading protocols on periimplant soft tissue health', Brazilian DentalScience. doi: 10.14295/bds.2020.v23i4.1998.

Schnitman, P. A. et al. (1997) 'Ten-year results for Brånemark implants immediately loaded with fixed prostheses at implant placement.', International Journal of Oral \& Maxillofacial Implants, 12(4).

van Steenberghe, D. et al. (1990) 'The Applicability of Osseointegrated Oral Implants in the Rehabilitation of Partial Edentulism: A Prospective Multicenter Study on 558 Fixtures.', International Journalof Oral \& Maxillofacial Implants, 5(3).

Tarnow, D. P., Emtiaz, S. and Classi, A. (1997) 'Immediateloading of threaded implants at stage 1 surgery in edentulous arches: ten consecutive case reports with 1-to 5-year data.', International Journal of Oral \& Maxillofacial Implants, 12(3).

Tettamanti, L. et al. (2017) 'Immediate loading implants: Review of the critical aspects', ORAL and 
Implantology. doi: 10.11138/orl/2017.10.2.129.

Thalib, B. et al. (2017) 'Differences of Post-Placement Bone Implant Contact (BIC) Value of Dental Implant Coated and Not Coated With Platelet Rich Plasma (PRP)', Global Journal of Health Science. doi:10.5539/gjhs.v10n2p11.

Xu, L. et al. (2014) 'Immediate versus early loading of flapless placed dental implants: A systematic review', Journal of Prosthetic Dentistry. doi: 10.1016/j.prosdent.2014.01.026.

Zhang, S., Wang, S. and Song, Y. (2017) 'Immediate loading for implant restoration compared with early or conventional loading: A meta-analysis', Journal of Cranio-Maxillofacial Surgery. doi: 10.1016/j.jcms.2016.05.002. 